\title{
Finding a Small Root of a Bivariate Integer Equation; Factoring with High Bits Known
}

\author{
Don Coppersmith \\ IBM Research \\ T.J. Watson Research Center \\ Yorktown Heights, NY 10598, USA
}

\begin{abstract}
We present a method to solve integer polynomial equations in two variables, provided that the solution is suitably bounded. As an application, we show how to find the factors of $N=P Q$ if we are given the high order $\left((1 / 4) \log _{2} N\right)$ bits of $P$. This compares with Rivest and Shamir's requirement of $\left((1 / 3) \log _{2} N\right)$ bits.
\end{abstract}

\section{Introduction}

We present a method to solve a polynomial equation $p(x, y)=0$ over $\mathbb{Z}$, provided that the solution is suitably bounded: $|x|<X$ and $|y|<Y$, with $X, Y$ depending on the coefficients and degree of $p$.

Our algorithm uses lattice basis methods [2]. It is similar in spirit to [1], which solved equations in one variable in $(\mathbb{Z} \bmod N)$, but the present algorithm requires a different analysis.

We require bounds $X$ and $Y$ on the absolute values of $x$ and $y$ in our solution. Suppose $p(x, y)$ has degree $\delta$ in each variable, and $p(x, y)=\sum_{i j} p_{i j} x^{i} y^{j}$. Define $D=\max _{i j}\left|p_{i j}\right| X^{i} Y^{j}$ as the largest possible term in $p(x, y)$ in the region of interest. Then we will find a bounded solution $(x, y)$ (if it exists) provided that

$$
X Y<D^{2 /(38)} .
$$

For fixed degree $\delta$, the algorithm runs in time polynomial in $(\log D)$.

Similar methods can be applied to the multivariate case but are not assured of success; the proof breaks down at a critical point.

Our immediate application, and the framework in which the algorithm is described, is the problem of factoring an integer when we know the high order bits of its factors. If we know $N=P Q$ and we know the high order $\left(\frac{1}{4} \log _{2} N\right)$ bits of $P$, then by solving the equation $\left(P_{0}+x\right)\left(Q_{0}+y\right)-N=0$ over a suitable range of $x$ and $y$ we can find the factorization of $N$. By comparison, Rivest and Shamir [5] need about $\left(\frac{1}{3} \log _{2} N\right)$ bits of $P$. This has applications to some RSA-based cryptographic schemes; see for example [7].

We give here a sket,ch of our algorithm. Define integer variables $r_{g h}$ representing $x^{g} y^{h}$. Form the lattice of those values of $\left\{r_{g h}\right\}$ satisfying several polynomial relations $q_{i j}(x, y)=x^{i} y^{j} p(x, y)=0$ under this interpretation. Claim that the latticc element $\mathbf{s}$ corresponding to our desired solution is relatively short (less U. Maurer (Ed.): Advances in Cryptology - EUROCRYPT '96, LNCS 1070, pp. 178-189, 1996. 
than the $n$th root of the determinant of a certain matrix). The expression of $\mathbf{s}$ in terms of a reduced basis of our lattice cannot involve the longest basis element (because $\mathbf{s}$ is short), so $\mathbf{s}$ is confined to the hyperplane spanned by the other basis elements. This gives a linear equation on $\left\{r_{g h}=x^{g} y^{h}\right\}$, which we interpret as a polynomial equation on $x$ and $y$. We combine this with $p(x, y)=0$ and solve for $x_{0}$ and $y_{0}$.

The remainder of the paper is organized as follows. In Section 2 the algorithm is developed, concentrating on the concrete case where $p(x, y)$ has degree 1 in each variable. Section 3 gives a brief discussion of linear lattice methods as applied to the nonlinear problem of solving polynomials. In Section 4 we extend the algorithm to other bivariate polynomials, and discuss the dependence on the size parameter $D$ and degree $\delta$ of the polynomial $p$. We comment on possible extensions to three or more variables in Section 5 . In Sections 6 and 7 we compare the current algorithm with previous ones. An application to Vanstone and Zuccherato's RSA variant is given in Section 8 . The appendix proves a technical result on Toeplitz matrices.

\section{Factoring with high order bits known}

We present the algorithm in terms of the problem of factoring an integer when we know the high-order bits of one of the factors.

Suppose we know $N=P Q$, and suppose that for sorne $\epsilon>0$ we know the high order $\left(\frac{1}{4}+\epsilon\right)\left(\log _{2} N\right)$ bits of $P$. (We will dispense with the $\epsilon$ later.) By division we know the high order bits of $Q$ as well.

$$
\begin{aligned}
P & =P_{0}+x_{0} \\
Q & =Q_{0}+y_{0} \\
\left|x_{0}\right| & <X=P_{0} / N^{(1 / 4)+c} \\
\left|y_{0}\right| & <Y=Q_{0} / N^{(1 / 4)+\epsilon} \\
p(x, y) & =\left(P_{0}+x\right)\left(Q_{0}+y\right)-N \\
& =\left(P_{0} Q_{0}-N\right)+Q_{0} x+P_{0} y+x y \\
p\left(x_{0}, y_{0}\right) & =P Q-N=0
\end{aligned}
$$

Here $P_{0}$ and $Q_{0}$ are known, while $x_{0}$ and $y_{0}$ are unknown, and $x$ and $y$ are dummy variables. $p(x, y)$ is an irreducible polynomial with integer coefficients, and its coefficients share no common factor.

We will relate the bounds $X$ and $Y$ to the quantity

$$
D=\max \left\{\left|P_{0} Q_{0}-N\right|, Q_{0} X, P_{0} Y, X Y\right\} .
$$

This is the largest possible size of an individual term of $p(x, y)$ with bounded $x$ and $y$. For our methods to work, we will require $(X Y)^{3 / 2}<D$. In the case of a more general polynomial $p(x, y)=\sum_{i j} p_{i j} x^{i} y^{j}$, of degree $\delta$ separately in each variable, we would define

$$
D=\max _{i j}\left\{\left|p_{i j}\right| X^{i} Y^{j}\right\}
$$


and demand $(X Y)^{3 \delta / 2}<D$. (The definitions of $X, Y, D$ appear circular, but it's all right; the condition is equivalent to existence of indices $i, j \leq \delta$ such that $\mathrm{X}^{(3 \delta / 2)-i} Y^{(3 \delta / 2)-j}<\left|p_{i j}\right|$, and the exponents $(3 \delta / 2)-i$ and $(3 \delta / \overline{2})-j$ are strictly positive.)

We are trying to find a bounded pair of integers $\left(x_{0}, y_{0}\right)$ solving $p\left(x_{0}, y_{0}\right)=0$. We begin by selecting an integer $k>1 /(4 \epsilon)$. For each pair of integers $(i, j)$ with $0 \leq i<k$ and $0 \leq j<k$, form the polynomial $q_{i j}(x, y)=x^{i} y^{j} p(x, y)$. Obviously $q_{i j}\left(x_{0}, y_{0}\right)=0$.

Form a matrix $M_{1}$ with $(k+1)^{2}$ rows, indexed by $\gamma(g, h)=(k+1) g+h$ with $0 \leq g, h<k+1 . M_{1}$ has $(k+1)^{2}+k^{2}$ columns, the left-hand columns indexed by $\gamma(g, h)$, and the right-hand columns indexed by $\beta(i, j)=(k+1)^{2}+k i+j$ with $0 \leq i, j<k$. The left-hand block is a diagonal matrix whose $(\gamma(g, h), \gamma(g, h))$ entry is given by $X^{-g} Y^{-h}$. The $(\gamma(g, h), \beta(i, j))$ entry of the right-hand block is the coefficient of $x^{g} y^{h}$ in the polynomial $q_{i j}(x, y)$.

An explanation of $M_{1}$ is in order. The $\gamma(g, h)$ row corresponds to an integer unknown $r_{g h}$ which represents $x_{0}^{g} y_{0}^{h}$. In the left-hand block, the diagonal entry $X^{-g} Y^{-h}$ will be used to bound $\left|r_{g h}\right|$ by approximately $X^{g} Y^{h}$. We will be concentrating on the sublatlice in which the right-hand columns are zero; a zero in columm $\beta(i, j)$ will correspond to the condition $q_{i j}\left(x_{0}, y_{0}\right)=0$.

Perform elementary row operations on $M_{1}$ to produce a matrix $M_{2}$ whose right-hand block has the $k^{2} \times k^{2}$ identity matrix on the bottom and the $(2 k+$ 1) $\times k^{2}$ zero matrix on the top. We can do this because the coefficient of $x y$ in $p$ is 1 , so that the right-hand block of $M_{1}$ contains an upper triangular matrix with 1 on the diagonal. (For a more general polynomial $p(x, y)$, we require that the coefficients of $p$ share no nontrivial common factor; in other words, $p(x, y)$ does not factor as $k \times u(x, y)$ over $\mathbb{Z}$.)

The lattice formed by these top $2 k+1$ rows of $M_{2}$ is the sublattice of the original lattice gotten by forcing to 0 all the right-hand columns. Call it $M_{3}$.

Consider the $(k+1)^{2}$-long row vector $\mathbf{r}$ whose $\gamma(g, h)$ entry is $x_{0}^{g} y_{0}^{h}$. T'he row vector $\mathbf{s}$ of length $(k+1)^{2}+k^{2}$ given by $\mathbf{s}=\mathbf{r} M_{1}$ satisfies

$$
\begin{aligned}
s_{\gamma(g, h)} & =\left(x_{0} / X\right)^{g}\left(y_{0} / Y\right)^{h} \\
\left|s_{\gamma(g, h)}\right| & \leq 1 \\
s_{\beta(i, j)} & =q_{i j}\left(x_{0}, y_{0}\right)=0 \\
|\mathbf{s}| & <k+1
\end{aligned}
$$

Because its right-hand side is $0, \mathbf{s}$ is one of the vectors in the lattice spanned by the rows of $M_{3}$. We will show that it is a "relatively short" vector in the lattice, which will enable us to confine it to a hyperplane, thus producing a linear equation relating its coefficients. This will translate directly to a polynomial equation on $x_{0}$ and $y_{0}: u\left(x_{0}, y_{0}\right)=0$, where $u(x, y)$ is not a multiple of $p(x, y)$. We can then take the resultant of $u(x, y)$ with $p(x, y)$ to find a single polynomial equation $v(x)=0$ satisfied by $x_{0}$, and solve this equation over $\mathbb{Z}$ to find $x_{0}$.

We proceed to estimate the sizes of the vectors in row lattice spanned by $M_{3}$, by estimating the determinant of a square submatrix of $M_{3}$. Define the diagonal matrix $W$ of dimension $(k+1)^{2} \times(k+1)^{2}$, with $(\gamma(g, h), \gamma(g, h))$ entry given 
by $X^{g} Y^{h}$. In the matrix $W M_{1}$, the left-hand block is the identity. In the $\beta(i, j)$ column of the right-hand side of $W M_{1}$, the largest element has absolute value $X^{i} Y^{j} D$. That is, the element at $(\gamma(g, h), \beta(i, j))$ is

$$
X^{g} Y^{h} p_{g-i, h-j}=X^{i+a} Y^{j+b} p_{a b}=X^{i} Y^{j}\left(X^{a} Y^{b} p_{a b}\right)
$$

where $a=g-i$ and $b=h-j$. Further, the right-hand columns are "nearly orthogonal", because they are part of a Toeplitz array. (A formal statement and proof appear in the appendix.) Associated with this near orthogonality, there is a specific set of $k^{2}$ columns in the left-hand block. Whenever we delete these $k^{2}$ columns from a rectangular matrix $M$, we denote the resulting squarc matrix as $M$. Deleting these columns from $W M_{1}$ leaves a matrix $W M_{1}$ whose determinant satisfies

$$
\begin{aligned}
\left|\operatorname{det}\left(W M_{1}\right)\right| & =\Omega\left(\prod_{i j}\left(X^{i} Y^{j} D\right)\right) \\
& =\Omega\left((X Y)^{k^{2}(k-1) / 2} D^{k^{2}}\right),
\end{aligned}
$$

the constant implicit in $\Omega$ depending on $k$ and the pattern of nonzero cocfficients in the polynomial $p(x, y)$, as shown in the appendix. For the polynomial in our example, the constant is 1 (see appendix) so we drop the " $\Omega$ ":

$$
\left|\operatorname{det}\left(W M_{1}\right)\right|=(X Y)^{k^{2}(k-1) / 2} D^{k^{2}} .
$$

Since

$$
\operatorname{det}(W)=\prod_{g h}\left(X^{g} Y^{h}\right)=(X Y)^{(k+1)^{2} k / 2}
$$

we can calculate

$$
\begin{aligned}
& \left|\operatorname{det}\left(M_{1}\right)\right|=\left|\operatorname{det}\left(W M_{1}\right)\right| / \operatorname{det}(W) \geq D^{k^{2}}(X Y)^{\left\{k^{2}(k-1) / 2\right\}-\left\{(k+1)^{2} k / 2\right\}} \\
& =D^{k^{2}}(X Y)^{-k(3 k+1) / 2}=\left(D^{k}(X Y)^{-(3 k+1) / 2}\right)^{k} \text {. }
\end{aligned}
$$

Remark. Here is where we will use the fact that $(X Y)^{3 / 2}<D$; in this example, it happens to be a consequence of the knowledge of $\left(\frac{1}{4}+\epsilon\right)\left(\log _{2} N\right)$ bits.

From

$$
\begin{array}{rlrl}
D & \geq\left|P_{0} Y\right| & =P_{0} Q_{0} / N^{1 / 4+\epsilon} \\
X Y & =\left(P_{0} / N^{1 / 4+\epsilon}\right)\left(Q_{0} / N^{1 / 4+c}\right)=P_{0} Q_{0} / N^{1 / 2+2 \epsilon}
\end{array}
$$

we see

$$
D^{k}(X Y)^{-(3 k+1) / 2} \geq\left(P_{0} Q_{0}\right)^{-(k+1) / 2} N^{(k / 2)+(1 / 4)+(2 k+1) \varepsilon} .
$$

Because $P_{0} Q_{0}=N\left(1+o\left(N^{-1 / 4}\right)\right)$ we can replace $P_{0} Q_{0}$ by $N$ and incur negligible error:

$$
D^{k}(X Y)^{-(3 k+1) / 2} \geq N^{(-1 / 4)+(2 k+1)}\left(1+o\left(k N^{-1 / 4}\right)\right) .
$$

Recall $k>1 /(4 c)$. Then

$$
\begin{gathered}
D^{k}(X Y)^{-(3 k+1) / 2}>N^{(+1 / 4)+\epsilon}\left(1+o\left(k N^{-1 / 4}\right)\right)>N^{1 / 4} . \\
\left|\operatorname{det}\left(M_{1}\right)\right|>N^{k / 4} \gg 1 .
\end{gathered}
$$


The same estimate applies to $\left|\operatorname{det}\left(M_{2}\right)\right|$ because $M_{2}$ was gotten from $M_{1}$ by elementary row operations. Also, because the lower right $k^{2} \times k^{2}$ submatrix of $\hat{M}_{2}$ is the identity and the upper right submatrix is zero, the same estimate applies to the determinant of the upper left $(2 k+1) \times(2 k+1)$ submatrix of $\hat{M}_{2}$, namely the left-hand $(2 k+1) \times(2 k+1)$ square submatrix of $M_{3}$. For the following discussion, we call that square submatrix $L$ and its dimension $n=2 k+1$. So $|\operatorname{det}(L)|>N^{k / 4}$.

We apply lattice basis reduction to the row basis of $L$, as prescribed in [2], to produce a reduced basis $\mathbf{b}_{1}, \mathbf{b}_{2}, \ldots, \mathbf{b}_{n}$. From the discussion in [2], the last element $\mathbf{b}_{n}$ of this reduced basis satisfies

$$
\left|\mathbf{b}_{n}^{*}\right| \geq|\operatorname{det}(L)|^{1 / n} 2^{-(n-1) / 4},
$$

where $\mathbf{b}_{n}^{*}$ denotes the component of $\mathbf{b}_{n}$ orthogonal to the subspace spanned by the vectors $\mathbf{b}_{1}, \ldots, \mathbf{b}_{n-1}$. As long as

$$
k<\frac{1}{4} \log _{2} N-2 \log _{2} \log _{2} N-\Omega(1)
$$

we will have that $\left|\mathbf{b}_{n}^{*}\right|>|\mathbf{s}|$ :

$$
\begin{aligned}
& \left|\mathbf{b}_{n}^{*}\right| \geq|\operatorname{det}(L)|^{1 / n} 2^{-(n-1) / 4}>N^{(k / 4)\left(1 /(2 k+1) / 2^{-2 k / 4}\right.}
\end{aligned}
$$

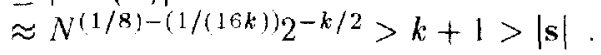

Assume this inequality holds. For any row vector $\mathbf{t}$ in the lattice spanned by $L$, if $\mathbf{t}$ is not in the lattice spanned by $\mathbf{b}_{1}, \ldots, \mathbf{b}_{n-1}$, then its expression as an integer conbination of the $\mathbf{b}_{i}$ involves $\mathbf{b}_{n}$ nontrivially. Thus we have $|\mathbf{t}| \geq$ $\left|\mathbf{b}_{n}^{*}\right|>k+1>|\mathbf{s}|$. Looked at the other way, for any $\mathbf{t}$ in the lattice spanned by $L$, if $|\mathbf{t}| \leq|\mathbf{s}|$, then $\mathbf{t}$ is in the lattice spanned by $\mathbf{b}_{1}, \ldots, \mathbf{b}_{n-1}$.

Consider s itself. The $n=2 k+1$ entries of $\mathrm{s}$ corresponding to those columns in the left-hand side that remain when we transform $M$ to $\hat{M}$, form a row vector $\mathbf{s}$ in the lattice spanned by $L$ (since the right-hand elements of $\mathbf{s}$ are 0 ). Also, $|\mathbf{s}| \leq|\mathbf{s}|$. Thus $\mathbf{s}$ is in the lattice spanned by $\mathbf{b}_{1}, \ldots, \mathbf{b}_{n-1}$.

Membership in this subspace gives us a linear relation on the coefficients $r_{g h}=x_{0}^{g} y_{0}^{h}$ expressing $\mathbf{s}$ as a linear combination of the rows of $M_{1}$. This relation is linearly independent of the $k^{2}$ relations given by the polynomials $q_{i j}(x, y)$ which determined that s had right-hand side 0 and thus was in the lattice of $M_{3}$ to start with. So this relation translates to a polynomial relation $u\left(x_{0}, y_{0}\right)=0$ where $u(x, y)$ is nol a polynomial multiple of $p(x, y)$.

Take the resultant of $p(x, y)$ and $u(x, y)$ with respect to $y$. Because $p(x, y)$ is irreducible and $u(x, y)$ is not a polynomial multiple of $p(x, y)$, we have that Resultant $_{y}(p(x, y), u(x, y))=v(x)$ is a nontrivial integer polynomial $v(x)$ in one variable $x$ satisfied by $x_{0}: v\left(x_{0}\right)=0$. Since $u(x, y)$ has degree at most $k$ in each variable and $p(x, y)$ has degree 1 in each variable, $v(x)$ has degree at most $2 k$. Solve $v(x)=0$ over $\mathbb{Z}$ to find a small number of candidates for $x_{0}$, namely those integer solutions satisfying the bound $\left|x_{0}\right|<X$.

Each casdidate value of $x_{0}$ can be substituted into $p$ to get an equation $p\left(x_{0}, y\right)=0$ which we can solve for $y$ over $\mathbb{Z}$, and select those integer solutions satisfying the bound $\left|y_{0}\right|<Y$. 
'Thus we have proven:

Theorem 1. If we know an integer $N=P Q$ and we know the high order $(1 / 4+$ $\epsilon)\left(\log _{2} N\right)$ bits of $P$, with $\epsilon>2 /\left(\log _{2} N\right)$, then in time polynomial in $\log N$ and $1 / \epsilon$ we can discover $P$ and $Q$.

Proof. The condition on $\epsilon$ insures that $k$ can be chosen to satisfy

$$
\frac{1}{4 \epsilon}<k<\frac{\log _{2} N}{4}-O(\log \log N)
$$

The complexity is due to invocation of lattice basis reduction on a matrix of size $2 k+1 \approx 1 /(2 c)$, whose elements are integcrs with bit length bounded by a polynomial in $\log N$. (We have to transform our rational matrices to integer matrices by multiplying by some integer.)

Corollary 2. If we know an integer $N=P Q$ and we know the high order $(1 / 4)\left(\log _{2} N\right)$ bits of $P$, then in time polynomial in $\log N$ we can discover $P$ and $Q$.

Proof. Set $c=4 / \log _{2} N$ and do exhaustive search on $O(1)$ unknown high order bits of $x_{0}$ (or middle bits of $P$ ).

\section{Discussion on lattice methods}

The lattice basis reduction method is inherently linear. If we want to relate several unknowns by a polynomial equation $p(x, y)=\sum_{g h} p_{g h} x^{g} y^{h}=0$, one natural approach is to replace each monomial $x^{g} y^{h}$ by a new independent variable $r_{g h}$, and let $p$ become a linear relation among several independent bounded variables: $\sum_{g h} p_{g h} r_{g h}=0,\left|r_{g h}\right| \leq X^{g} Y^{h}=R_{g h}$. In order to get results, we would need the desired vector to be among the shortest of the lattice; we would need its length to be smaller than the root of the determinant of the appropriate square matrix. 'This requirement translates to (approximately) $\prod R_{g h} \leq D$, and this would imply severe restrictions on $X$ and $Y$, namely $(X Y)^{(\delta+1)^{2} \delta / 2} \leq D$, where $p(x, y)$ has degree $\delta$ in each variable. This is essentially the technique used by [6] in the modular setting.

In the present paper we have extended this approach by using several polynomials $q_{i j}$ but reusing the same independent bounded variables $r_{g h}$. We are able to amortize the cost of the several variables over several equations. This accounts for the success of the present method in increasing the feasible sizes of $X$ and $Y$. Specifically, each unknown $r_{g h}$ contributes a factor of $X^{-g} Y^{-h}$ to $\operatorname{det}\left(M_{1}\right)$, while each equation $q_{i j}(x, y)=0$ contributes a factor of $X^{i} Y^{j} D$. In order for our techniques to work, we require $\operatorname{det}\left(M_{1}\right) \gg 1$, which yields a bound on $\mathrm{X}$ and $\mathrm{Y}$ in terms of $D$. Because we have several polynomial equations, each contributing positively to the determinant, this bound is relatively mild; we can tolerate larger ranges $X, Y$ on our variables (in terms of $D$ ) than with other methods, namely $(X Y)^{38 / 2}<D$, as we will see in the next section. 


\section{Other bivariate polynomials}

Similar techniques can be applied successfully to other polynomial equations besides the given $p(x, y)=0$. They are not guaranteed for polynomials of more than two variables; see Section 5.

Fven in the case of two variables, the present technique is sensitive to the form of the polynomial $p(x, y)$. For an arbitrary quadratic polynomial we could not tolerate ranges $X, Y$ for the unknowns $x, y$ quite as large as wo did here. In the case that we used for our example, although $\nu(x, y)$ has degree two, it does not have terms involving $x^{2}$ or $y^{2}$, only $x y$.

We sketch here how the bounds $X$ and $Y$ depend on $D$ and the form of $p$. When we estimated $\left|\operatorname{det}\left(M_{1}\right)\right|$ by $\operatorname{dividing}$ the estimate of $\left|\operatorname{det}\left(W M_{1}\right)\right|$ by det $(W)$, there was considerable cancellation in the powers of $X$ and $Y$. The term $\left|\operatorname{det}\left(W M_{1}\right)\right|$ had a factor $X^{i} Y^{j} D$ for cach pair $(i, j)$ with $0 \leq i, j<k$; these pairs represented the monomials $x^{i} y^{j}$ by which $p(x, y)$ was multiplied. The term $\operatorname{det}(W)$ had a factor $X^{g} Y^{h}$ for each pair $(g, h)$ with $0 \leq g, h<k+1$; these pairs rcpresented the monomials $x^{g} y^{h}$ appearing in these products $x^{i} y^{j} p(x, y)$. The range on $g$ exceds the range on $i$ by 1 because $p$ has degree 1 in $x$. If $p$ had an $x^{2}$ term, we would have needed to enlarge the range of $g$. The powers of $X$ and $Y$ appearing in the ratio $\left|\operatorname{det}\left(W \dot{M}_{1}\right)\right| / \operatorname{det}(W)$ arise from the pairs $(g, h)$ outside the range of $(i, j)$, namely

$$
\{(g, h) \mid g=k, 0 \leq h<k\} \cup\{(g, h) \mid h=k, 0 \leq g \leq k\}
$$

The inclusion of an $x^{2}$ term would have enlarged that region by another layer. This would have led directly to a stricter requirement on the sizes of $X$ and $Y$.

If our polynomial $p(x, y)$ has degree $\delta$ in $x$ and $\tau$ in $y$, then we can tolerate ranges $X$ and $Y$ satisfying

$$
X^{\delta+(\tau / 2)} Y^{\tau+(\delta / 2)}<D
$$

by using polynomials $q_{i j}(x, y)$ in the range $0 \leq i, j<k$. More generally, for any positive value of the parameter $\alpha$ we can tolerate $X$ and $Y$ with

$$
\left.X^{\delta+(\alpha \tau / 2)}\right)^{\tau+(\delta /(2 \alpha))}<D
$$

by allowing $0 \leq i<k \alpha$ and $0 \leq j<k$.

If $p(x, y)$ has total degree $\delta$ then we can tolerate about

$$
D>(X Y)^{\delta}
$$

by allowing pairs $(i, j)$ with $i \geq 0, j \geq 0$, and $i+j<k$. This is better than the previous approach if $p$ is a general bivariate polynomial of total degree $\delta$, but worse if (like the current example) it is really of degree $\delta / 2$ separately in cach variable.

We sunnlarize these results: 
Theorem 3. Let $p(x, y)=\sum_{i j} p_{i j} x^{i} y^{j}$ be a bivariate polynomial over $\mathbb{Z}$ of degree $\delta$ in $x$ and $\tau$ in $y$. Assume $p$ is irreducible over $\mathbb{Z}$. Let $X$ and $Y$ be bounds on desired solutions $\left|x_{0}\right|$ and $\left|y_{0}\right|$. Define $D=\max _{i j}\left|p_{i j}\right| X^{i} Y^{j}$. Choose $\alpha>0$. Assume

$$
X^{\delta+(\alpha \tau / 2)} Y^{\tau+(\delta /(2 \alpha))}<D \times 2^{-3\left(\delta^{2}+\tau^{2}\right)-2}
$$

In time polynomial in $\delta, \tau$ and $\log _{2} D$, our algorithm will produce all integer pairs $\left(x_{0}, y_{0}\right)$ with $\left|x_{0}\right|<X,\left|y_{0}\right|<Y$, and $p\left(x_{0}, y_{0}\right)=0$.

Let $p(x, y)$ be as before, but with total degree $\delta$. Assume

$$
(X Y)^{\delta}<D \times 2^{-6 \delta^{2}-2}
$$

In time polynomial in $\delta$ and $\log _{2} D$, our algorithm will produce all integer pairs $\left(x_{0}, y_{0}\right)$ wilh $\left|x_{0}\right|<X,\left|y_{0}\right|<Y$, and $p\left(x_{0}, y_{0}\right)=0$.

Proof. The proof will be given in the full paper, but is quite similar to that of Theorem 1 and Corollary 2.

\section{More variables}

Suppose we have a polynomial $p(x, y, z)$ in three variables. We can mimic the present approach. If the ranges $X, Y, Z$ are small enough, we will end up with a polynomial relation $u(x, y, z)$, not a multiple of $p$, satisfied by $\left(x_{0}, y_{0}, z_{0}\right)$. Then the resultant of $p$ and $u$ with respect to $z$ will give a polynomial $v(x, y)$ in two variables. We can then try to solve $v$ by the current methods. But the degree of " will bo quite high, so that the ranges $X$ and $Y$ which can be tolerated will be quite small. This approach will be unsatisfactory in general.

We still have a heuristic procedure which might work for a given multivariate polynomial. We are guaranteed to find a space of codimension 1 (a hyperplane) containing all the short vectors of the lattice $M_{3}$. But we might easily find a space of larger codimension. (There is a good possibility that for many basis vectors $\mathbf{b}_{i}$, the orthogonal component $\left|\mathbf{b}_{i}^{*}\right|$ exceeds our known upper bound on $|s|$, and each one increases the codimension of the space which contains all the short vectors.) We develop several polynomial equations $u_{i}(x, y, z)$ satisfying $u_{i}\left(x_{0}, y_{0}, z_{0}\right)=0$; the number of such equations is equal to the codimension of this space. We can then take resultants and gcd's of the various $u_{i}$ and $p$ and hope to produce a single polynomial equation in a single variable $v\left(x_{0}\right)=0$, which we solve over $\mathbb{Z}$. 'This is only a heuristic approach, which might or might not work for a given polynomial $p$. (Even if we can generate several equations, they may not be independent.)

There must be linits to the success of this approach in general. Manders and Adleman [3] show that finding suitably bounded solutions to $p_{N}(x, y, z)=$ $x^{2}-y N-z=0$ is NP-hard. Nonetheless the approach might work for a particular polynomial, and it is worth trying. 


\section{Comparison with the univariate modular algorithm}

In a companion paper [1], the author applies a very similar algorithm for the solution of a univariate modular polynomial.

'T'wo differences between the algorithms are worth noticing. In the modular case, the size $X$ of the acceptable solutions $x_{0}$ was related to the modulus $N$. In the present integer case, there is no such natural measure as $N$, and we needed to develop a bound in terms of $D$.

A second difference is that in the modular case, we were able to define polynomials $q_{i j}(x)=x_{i}^{i} p(x)^{j}$ and assert that $q_{i j}\left(x_{0}\right)=0 \quad\left(\bmod N^{i}\right)$; the extra information $\left(\bmod N^{j}\right.$ versus $\left.\bmod N\right)$ improved our bound on $X$ from $N^{1 /(2 \delta-1)}$ to $N^{1 / \delta}$. In the present integer case, using the polynomial equations $q_{i j k}(x, y)=$ $x^{i} y^{j} p(x, y)^{k}=0$ would not help, because (for the appropriate ranges of indices $i, j, k$ ) the integer linear combinations of the polynomials $q_{i j}$ are exactly the same as those of the polynomials $q_{i j k}$. For example, with the given $p(x, y), \delta=1$, and setting $k=4$, the integer linear combinations of

$$
q_{i j}(x, y)=x^{i} y^{j} p(x, y), \quad 0 \leq i, j<4
$$

are the same as the integer linear combinations of

$$
p, x p, x^{2} p, x^{3} p, y p, y^{2} p, y^{3} p, p^{2}, x p^{2}, x^{2} p^{2}, y p^{2}, y^{2} p^{2}, p^{3}, x p^{3}, y p^{3}, p^{4},
$$

so that we and up defining the same matrix $M_{1}$ (up to elementary column operations). (If $p$ is not monic, we appear to gain something from the high coefficicnt of $p$, but we actually lose a corresponding amount in the proof, so that using powers of $p$ still neither helps nor hurts us.) This much is also true in the modular case; however, there we gain the extra advantage of working $\bmod N^{j}$ as opposed to working $\bmod N$, and here in the integer case we can derive no such advantage.

\section{Comparison with previous work}

Rivest and Shamir [5] solve the problem of factorization if given $\left(\log _{2} N\right) / 3$ bits rather than $\left(\log _{2} N\right) / 4$ bits as we do. They too use lattice methods, but only one polynomial $q_{00}(x, y)=p(x, y)$.

Vallée et al. [6] employ a method similar to [5] in the case of modular polynomials, again using only one polynomial.

Maurer [4] uses a different approach, related to factoring algorithms based on smooth integers, to ask ( $c \log _{2} N$ ) yes/no oracle questions and determine the factorization of $N$ with failure probability $O\left(N^{-\epsilon / 2}\right)$.

\section{Application to RSA variant}

Vanstone and Zuccherato [7] propose an identity-based variant of RSA in which the user's modulus $N$ is related to his identity. For example, the high order bits of $N$ may be the user's name encoded in ASCII. 
Unfortunately, the modulus $N$ is generated in such a way that somewhat more than the high order $\left((1 / 4) \log _{2} N\right)$ bits of $P$ are revealed to the public. This enables the prosent attack to discover the factorization of each modulus and break the scheme.

\section{Acknowledgements}

Matt Franklin and Mike Reiter's Crypto 95 rump session talk opened this whole line of investigation; subsequent discussions were also useful. The author gratefully acknowledges enlightening discussions with Andrew Odlyzko. Barry Trager helped the experimental effort by coding up in Axiom an implementation of the Lenstra Lenstra Lovasz lattice basis reduction algorithm. The Eurocrypt program committee made suggestions which improved the presentation of the paper.

\section{References}

1. D. Coppersmith, "Finding a Small Root of a Univariate Modular Equation," Proceedings of Eurocrypt 96.

2. A. K. Lenstra, II. W. Lenstra and L. Lovasz, "Factoring Polynomials with Integer Coefficient.s," Matematische Annalen 261 (1982), 513-534.

3. K. Manders and L. Adleman, "NP-complete decision problems for binary quadratics," J. Comput. System Sci. 16, 168-184.

4. U. M. Maurer, "Factoring with an Oracle," Advances in Cryptology - EUROCRYP'T '92, Springer LNCS 658 (1993) $429 \cdots 436$.

5. R. L. Rivest and A. Shamir, "Efficient factoring based on partial information," Advances in Cryptology - EUROCRYP'T '85, Springer LNCS 219 (1986) 31-34.

6. B. Vallée, M. Girault and P. Toffin, "How to Guess $\ell-$ th Roots Modulo n by Reducing Lattice Bases," Procecdings of AAECC; 6, Springer LNCS 357 (1988) 427-442.

7. S. A. Vanstone and R. J. Zuccherato, "Short RSA Keys and Their Generation," Journal of Cryptology 8 number 2 (Spring 1995) 101-114.

\section{Appendix}

In this appendix we give a proof of the technical result needed in Section 2: that several columns of the matrix $W M_{1}$ are "nearly orthogonal". A modification of this proof would apply to any Toeplitz matrix.

We develop the corresponding result for a general bivariate polynomial. Let $M_{4}$ be the right-hand block of $W M_{1}$. Let $p(x, y)$ have degree $\delta$ in each variable separately. Define indexing functions $\gamma$ and $\mu$ for $M_{4}$ : 'The rows of $M_{4}$ are indexed by $\gamma(g, h)=(k+\delta) g+h$ for $0 \leq g, h<k+\delta$, and the columns by $\mu(i, j)=k i+j$ for $0 \leq i, j<k$. Define $\tilde{p}(x, y)=p(X x, Y y)$, so that $\dot{p}_{a b}=X^{a} Y^{b} p_{a b}$ and $\max _{a b}\left|p_{a b}\right|=D$. 
Lemma 4. There is a $k^{2} \times k^{2}$ submatrix of $M_{4}$ whose determinant has absolute value at least

$$
D^{k^{2}} 2^{-6 k^{2} \delta^{2}-2 k^{2}}
$$

If the largest coefficient of $\tilde{p}$ is one of $\tilde{p}_{00}, \tilde{p}_{0 \delta}, \tilde{p}_{\delta_{0}}$ or $\tilde{p}_{\delta \delta}$, then the absolute value of the determinant is exactly $D^{k^{2}}$.

Proof. Select indices $(a, b)$ so that $D=\left|\bar{p}_{a b}\right|$ is the largest coefficient of $\tilde{p}$. Select indices $(c, d)$ to maximize the quantity

$$
8^{(c-a)^{2}+(d-b)^{2}}\left|j_{c d}\right|
$$

Select the rows

$$
\gamma(c+i, d+j), 0 \leq i, j<k
$$

of $M_{4}$ to create the desired submatrix $\tilde{M}$. The rows and columns of $\tilde{M}$ are indexed by $\mu(i, j)=k i+j$. The matrix elmment $\tilde{M}_{\mu(g, h !, \mu(i, j)}$ is the coefficient of $x^{c+g} y^{d+h}$ in $x^{i} y^{j} \tilde{p}(x, y)$, namely

$$
\grave{M}_{\mu(g, h), \mu(i, j)}=\tilde{p}_{g-i+c, h-j+d}
$$

Multiply the $\mu(g, h)$ row of $\dot{M}$ by $8^{2(c-a) g+2(d-b) h}$, and multiply the $\mu(i, j)$ column by $8^{-2(e-a) i-2(d-b) j}$, to create a new matrix $M^{\prime}$ with the same determinant. Its typical element is

$$
M_{\mu(g, h), \mu(i, j)}^{\prime}=\ddot{p}_{g-i+c, h-j+d} 8^{2(r-a)(g-i)+2(d-b)(h-j)} .
$$

From maximality of $(c, d)$ we find

$$
\left|\bar{p}_{g-i+c, h-j+d}\right| 8^{(g-i+c-a)^{2}+(h-j+d-b)^{2}} \leq\left|\tilde{p}_{c d}\right| 8^{(c-a)^{2}+(d-b)^{2}},
$$

from which

$$
\left|\tilde{p}_{g-i+c, h-j+d}\right| 8^{2(g-i)(c-a)+2(h-j)(d-b)} \leq\left|\bar{p}_{c d}\right| 8^{-(g-i)^{2}-(h-j)^{2}} .
$$

Thus cach diagonal entry of $M^{\prime}$ is $\hat{p}_{c d}$, and each off-diagonal cntry is bounded in absolute value by $\left|\tilde{p}_{c d}\right| 8^{-(g-i)^{2}-(h-j)^{2}}$. This implies that $M^{\prime}$ is diagonally dominant, because the absolute values of the off-diagonal entries in its $\mu(i, j)$ row sum to at most

$$
\begin{aligned}
& \left|\dot{p}_{c d}\right| \times \sum_{(g, h) \neq(i, j)} 8^{-(a-j)^{2}-(h-j)^{2}}=\left|\tilde{p}_{c d}\right| \times \sum_{(a, b) \neq(0,0)} 8^{-a^{2}-b^{2}} \\
& =\left|\dot{p}_{c d}\right| \times\left[-1+\sum_{(a, b)} 8^{-a^{2}-b^{2}}\right]=\left|\tilde{p}_{c d}\right| \times\left[-1+\left(\sum_{a b} 8^{-a^{2}}\right)^{2}\right]<\frac{3}{4}\left|\tilde{p}_{c d}\right|
\end{aligned}
$$

Each eigenvalue of $M^{\prime}$ is within $\frac{3}{4}\left|\tilde{p}_{c d}\right|$ of $\tilde{p}_{c d}$, and so exceeds $\frac{1}{4}\left|\tilde{p}_{c d}\right|$ in absolute value. By choice of $(c, d)$ we know

$$
\begin{gathered}
8^{(c-a)^{2}+(d-b)^{2}}\left|\dot{p}_{c d}\right| \geq 8^{0}\left|\tilde{p}_{a b}\right|=D \\
\left|\tilde{p}_{c a}\right| \geq 8^{-2 \delta^{2}} D
\end{gathered}
$$




$$
\left|\operatorname{det}\left(M^{\prime}\right)\right| \geq\left(\frac{1}{4}\left|\hat{p}_{c \cdot d}\right|\right)^{k^{2}} \geq\left(\frac{1}{4} 8^{-2 x^{2}} D\right)^{k^{2}}=D^{k^{2}} 2^{-6 k^{2} \delta^{2}-2 k^{2}} .
$$

For the second claim of the lemma: If the largest coefficient of $\tilde{p}$ is either $\tilde{p}_{00}$ or $\tilde{p}_{s \delta}$, set $(c, d)=(a, b)$ and notice that $\tilde{M}$ is an (upper or lower) triangular matrix whose diagonal cntries have absolute value $D$. If the largest coefficient is either $\hat{p}_{0 \delta}$ or $\dot{p}_{\delta 0}$, redefine the indexing function on columns as $\mu(i, j)=k i+(k-1-j)$ so that again $\dot{M}$ is a triangular matrix whose diagonal entries have absolute value $D$. Similar results hold if $(a, b)$ is any corner of the Newton polygon associated with $\bar{p}$.

For the particular case $p(x, y)=\left(P_{0}+x\right)\left(Q_{0}+y\right)-N$, we have $\delta=1$, and the only non-zero coefficients of $\tilde{p}$ are $\tilde{p}_{00}, \tilde{p}_{0 \delta}, \tilde{p}_{\delta 0}$ and $\tilde{p}_{\delta \delta}$; thus the second claim must hold.

The lemma gives a $k^{2} \times k^{2}$ submatrix $\bar{M}$ of $M_{4}$, where $M_{4}$ is the right-hand $(k+\delta)^{2} \times k^{2}$ block of $W M_{1}$. To apply the lemma, we need to find $k^{2}$ column indices in the lcft-hand block of $W M_{1}$ whose deletion leaves a $(k+\delta)^{2} \times(k+\delta)^{2}$ submatrix $W M_{1}$ of $W M_{1}$ with $\left|\operatorname{det}\left(W M_{1}\right)\right|=|\operatorname{det}(\hat{M})|$. We simply delete those columns whose indices match those of the $k^{2}$ rows accepted in $\tilde{M}$. Recall that the left-hand side of $W M_{1}$ is the identity matrix, so each remaining left-hand column has a single 1 among the 0 's, and expansion by minors gives $\left|\operatorname{det}\left(W \hat{M}_{1}\right)\right|=$ $|\operatorname{det}(\tilde{M})|$ as desired. 